\title{
A Case of Primary Gastric Melanoma Exhibiting a Rare BRAF V600R Mutation
}

\author{
Grainne M. Callaghan ${ }^{1}$, Fergal C. Kelleher ${ }^{2}$, Paul F. Ridgway ${ }^{3,4}$, Stephen Crowther ${ }^{5}$, Alaa Alakkari ${ }^{1}$, Barbara M. Ryan $^{1,6}$ \\ ${ }^{1}$ Department of Gastroenterology, Tallaght Hospital, Dublin, Ireland \\ 2 Department of Medical Oncology, Tallaght Hospital, Dublin, Ireland \\ ${ }^{3}$ Department of Surgery, Tallaght Hospital, Dublin, Ireland \\ ${ }^{4}$ Department of Surgery, Trinity College Dublin, Ireland \\ ${ }^{5}$ Department of Cellular Pathology, Tallaght Hospital, Dublin, Ireland \\ ${ }^{6}$ Department of Clinical Medicine, Trinity College Dublin, Ireland
}

Received: 26/09/2017

Accepted: 20/11/2017

Published: 03/01/2018

How to cite this article: Callaghan GM, Kellher FC, Crowther S, Alakkari A, Ryan BM. A case of primary gastric melanoma exhibiting a rare BRAF V600R mutation. EJCRIM 2018;5: doi:10.12890/2018_000749.

Conflicts of Interests: The Authors declare that there are no competing interests.

This article is licensed under a Commons Attribution Non-Commercial 4.0 License

\section{ABSTRACT}

Introduction: Malignant melanoma of the gastrointestinal tract is usually a metastasis from a cutaneous source. Primary gastric melanoma is an extremely rare clinical entity, with few reported cases worldwide. It is often advanced at time of diagnosis and is associated with a dismal outcome.

Background: A 76 year old gentleman presenteded with a one month history of fatigue and exertional dyspnoea. Laboratory investigations indicated an anaemia, with a haemoglobin level of $11.0 \mathrm{~g} / \mathrm{dl}$. Subsequent gastroscopy visualised a large, atypical, crater-like ulcerated lesion distal to the cardia in the proximal stomach.

Provisional histology was suggestive of a poorly differentiated adenocarcinoma but subsequent cyto-morphology and immunophenotyping were consistent with melanoma, with positive S100 protein, HMB45 and Melan A. Further molecular genetic testing revealed a V600R mutation in the BRAF gene, which is the first primary gastric melanoma with this mutation to be reported in the literature. Given the rarity of the findings, an extensive secondary work-up was undertaken, which concluded the diagnosis primary gastric melanoma.

Discussion: Primary gastric melanoma is a rare disease that can present similarly to other upper gastrointesinal lesions, with weight loss, abdominal pain, malena, and anaemia. Given its rarity, the pathogenesis is poorly understood. Lesions are often endoscopically atypical. Important points to note would include the absence of a primary lesion, as supported by a full skin examination and PET-CT findings, which can help to delineate the limitation to the stomach, thus helping to inform subsequent management.

\section{LEARNING POINTS}

- Primary gastric melanoma (PGM) is a rare clinical entity.

- Work-up including skin and ophthalmic examination is important to exclude a primary cutaneous source, as this helps dictate both prognosis and subsequent management, including whether surgical resection is advisable.

- Immunophenotyping and genetic testing inform management but, despite advances in therapy, the prognosis of PGM and other mucosal melanomas remains poor.

\section{KEYWORDS}

Gastric melanoma, malignant melanoma, BRAF 


\section{INTRODUCTION}

Primary gastric melanoma (PGM) is extremely rare, with few reported cases. PGM tends to metastasise early and has a poor outcome. We present the case of a patient whom an extensive work-up concluded PGM, with a rare BRAF V600R mutation. While BRAF mutations are common in cutaneous melanoma ${ }^{[1]}$, they are rare in mucosal melanomas, and have not previously been described in a PGM.

\section{CASE DESCRIPTION}

A 76-year-old male patient presented with a one-month history of fatigue, exertional dyspnoea and weight loss. He had iron deficiency anaemia. At gastroscopy, there was a large ulcerated lesion in the proximal stomach (Fig. 1). Histological examination revealed extensive lamina propria infiltration by a population of large epithelioid malignant cells. Immunohistochemistry was positive for S100 Protein, HMB45, and Melan-A, consistent with malignant melanoma. Further genetic analysis revealed a V600R mutation at exon 15 of the BRAF gene. Work-up, including a full skin examination and ophthalmology review, did not identify another primary source, and no metastatic disease was identified on radiological imaging. The consensus diagnosis of the treating physicians and surgeons was that findings were consistent with PGM. Subsequently, he underwent an open sleeve gastrectomy, and histology confirmed a melanomatous process, with two positive local lymph nodes.

Following post-operative recovery and prior to commencement of systemic treatment, a repeat CT scan of the thorax, abdomen and pelvis showed interval progression of the disease in the two-month interval, with new liver metastases and an enlarging left upper quadrant deposit. The patient commenced immunotherapy, but unfortunately died of disease progression within a few months.

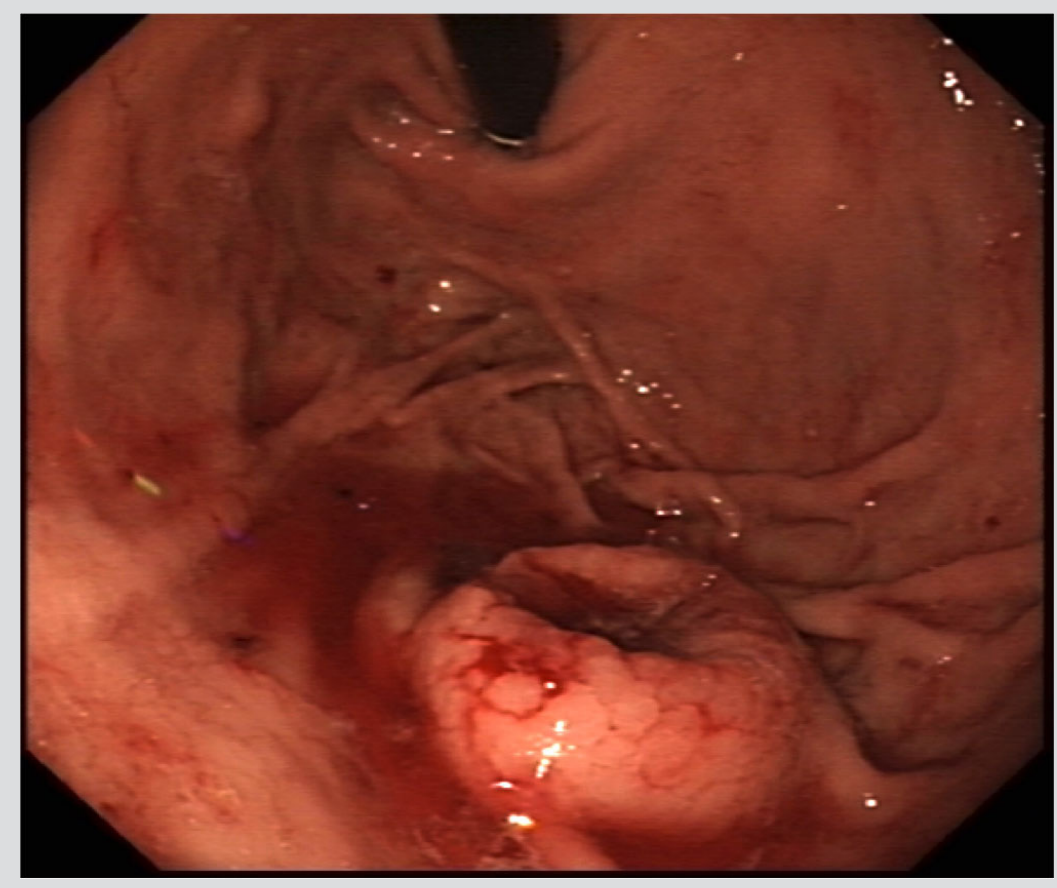

Figure 1. Endoscopic image of a large ulcerated lesion in the proximal stomach

\section{DISCUSSION}

Melanoma is an aggressive neoplasm that usually occurs in cutaneous sites, but can also arise from other less common sites such as uveal tracts and mucosa. Mucosal melanoma accounts for only $1.3 \%$ of all these cases, tends to present with advanced disease, and generally has a very poor prognosis ${ }^{[2]}$. PGM, a subtype of mucosal melanoma, is an even rarer occurrence, with only sporadic case reports in the literature ${ }^{[3]}$. PGM presents similarly to other upper gastrointestinal lesions, with weight loss, abdominal pain, and melaena. If histology is diagnostic of melanoma, it is important to exclude metastasis from a primary cutaneous or ocular lesion, with full skin examination by a dermatologist and an ophthalmological assessment to outrule another primary source, as this influences both the prognosis and further management, including whether surgical resection is advisable. Detailed radiological imaging is performed to delineate the burden of metastases, if indicated by histology. 
Genetic testing of melanoma is mandatory as it informs the treatment paradigm, with targeted therapies available for patients exhibiting specific mutations. Ninety per cent of all mutations are acquired, due to the effects of cumulative environmental factors, such as exposure to ultraviolet light. Mucosal melanomas have been found to exhibit different genetic mutations to their cutaneous counterparts, suggesting differing tumour biology ${ }^{[2]}$. Approximately $50 \%$ of cutaneous melanomas have a BRAF mutation ${ }^{[4]}$, whereas BRAF mutations are rare in mucosal melanomas ${ }^{[1]}$. The BRAF oncogenic effect is exerted through increased activation of the MAP kinase pathway, leading to uncontrolled cell proliferation ${ }^{[5]}$. Several BRAF mutations have been described, with V600E accounting for over $80 \%$ of mutations ${ }^{[4]}$. The V600R mutation (a substitution of arginine for valine at codon 600 of the BRAF gene) was identified in this patient, and accounts for approximately $4 \%$ of all melanoma $\operatorname{cases}^{[6]}$, and, to our knowledge, has not been previously described in a PGM.

There is no clear consensus on the management of PGM. In this case, the disease progressed rapidly despite management with surgery and immunotherapy, consistent with the scanty literature which suggests a uniformly poor prognosis.

\section{REFERENCES}

1. Wong CW, Fan YS, Chan TL, Chan AS, Ho LC, Ma TK, et al. BRAF and NRAS mutations are uncommon in melanomas arising in diverse internal organs. J Clin Pathol 2005;58:6404.

2. Dominiak NR, Wick MR, Smith MT. Mucosal melanomas: site-specific information, comparisons with cutaneous tumors, and differential diagnosis. Semin Diagn Pathol 2016:33:191-7.

3. Wang L, Zong L, Nakazato H, Wang WY, Li CH, Shi YF, et al. Primary advanced esophago-gastric melanoma: a rare case. World J Gastroenterol 2016; $22: 3296-3301$.

4. Ascierto PA, Kirkwood JM, Grob JJ, Simeone E, Grimaldi AM, Maio M, et al. The role of BRAF V600 mutation in melanoma. J Transl Med 2012;10:85.

5. Wellbrock C, Hurlstone A. BRAF as therapeutic target in melanoma. Biochem Pharmacol 2010;80:561-7.

6. Lovly CM, Dahlman KB, Fohn LE, Su Z, Dias-Santagata D, Hicks DJ, et al. Routine multiplex mutational profiling of melanomas enables enrollment in gentotype-driven therapeutic trials. PLOS One, 2012;7:e35309. 